\title{
ELLIPSE DETECTION USING SAMPLING CONSTRAINTS
}

\author{
Yi Tang and Sargur N. Srihari
}

\author{
Center of Excellence for Document Analysis and Recognition \\ University at Buffalo, The State University of New York \\ Amherst, New York 14228, U.S.A
}

\begin{abstract}
The ellipse is a fundamental shape in both natural and manmade objects and hence frequently encountered in images. Existing ellipse detection algorithms, viz., randomized Hough transform (RHT) and multi-population genetic algorithm (MPGA), have disadvantages. The RHT performs poorly with multiple ellipses and MPGA has a high false positive for complex images. The proposed algorithm selects random points using constraints of smoothness, distance and curvature. In the process of sampling, parameters of potential ellipses are progressively learnt to improve parameter accuracy. New probabilistic fitness measures are used to verify ellipses extracted: ellipse quality based on the Ramanujan approximation and completeness. Experiments on synthetic and real images show performance better than RHT and MPGA in detecting multiple, deformed, full or partial ellipses in the presence of noise and interference.
\end{abstract}

Index Terms - ellipse detection, randomized Hough transform, probabilistic fitness, footwear print

\section{INTRODUCTION}

The ellipse is a fundamental shape in images of both natural and man-made objects. Formed by the intersection of a cone and a plane, ellipses are useful to analyze and synthesize cylindrical objects on two dimensional planes. They describe planetary motion and effectively used by artists in creating realistic images. Some applications of ellipse detection in image processing are cell image segmentation [1], recognition of industrial parts [2] and analysis of footwear impressions [3].

Existing algorithms for ellipse detection are of three types: Hough transform (HT), Genetic algorithms (GA) and edge following. The Standard Hough transform (SHT) [4] detects parametric curves by mapping from image space to parameter space. In the case of straight line HT with two parameters each point is mapped to a curve in parameter space; thus it is a one-to-many diverging mapping which is slow and memory intensive. The situation gets worse for

Project supported by Award No. 2007-DN-BX-K135 by NIJ-OJP- U.S. DoJ; opinions expressed are those of the authors and not the DoJ. ellipses with five parameters. While the Randomized Hough transform (RHT) [5] alleviates these problems using a manyto-one converging mapping [6], its performance degrades as the number of ellipses in the image increases $[1,7,8]$. The second group, exemplified by the multi-population genetic algorithm (MPGA) [1], simulates the search for ellipses in feature space as a parallel optimization process. While it is better than RHT in accuracy and speed, because of its optimization criteria it may lead to local sub-optimal solutions. The third group, edge-following algorithms [8, 2], which extract ellipses using a hierarchical approach and perform well in handling occluded and overlapping ellipses, are susceptible to edge distortion [2] or fail when ellipses are linked to other curves seamlessly. While improvements of RHT have been proposed, e.g. [9, 7], they are still unsatisfactory for detecting multiple, potentially deformed, full or partial ellipses with noise and interference. In this paper the sampling process is improved by exploiting constraints, and allowing the sampler to learn the parameters of the potential ellipses which guide the selection of good samples. Two probabilistic fitness measures, weighted quality and completeness, are proposed to validate the extracted raw ellipses.

\section{CONSTRAINED RHT}

In the Cartesian $x, y$ plane, an ellipse is defined by $A x^{2}+$ $B x y+C y^{2}+D x+E y+F=0$ with $4 A C-B^{2}>0$. In parametric form used by SHT, the ellipse is described in terms of center $(p, q)$, length of semi-major/minor axes $a, b$ and orientation $\theta$ by

$$
\frac{((x-p) \cos \theta+(y-q) \sin \theta)^{2}}{a^{2}}+\frac{((y-q) \cos \theta-(x-p) \sin \theta)^{2}}{b^{2}}=1 .
$$

SHT is deterministic, computationally expensive and inaccurate as it uses a diverging mapping and requires a 5-D accumulator. RHT [5] is randomized, improves efficiency and accuracy by sampling followed by a converging mapping. It selects as many points as the number of parameters $n$ via uniform sampling ( $n=5$ for ellipse) and maps them into one point in parameter space, from which individual counts are accumulated to form peaks that correspond to ellipses. Ellipse geometry can be used to reduce complexity and improve 


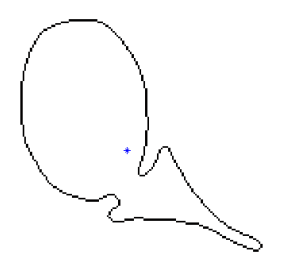

(a) A deformed ellipse

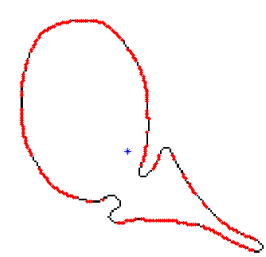

(b) SC only

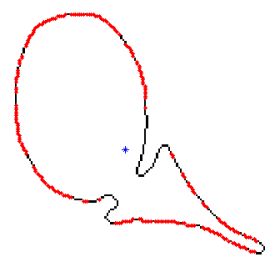

(c) SC \& DC

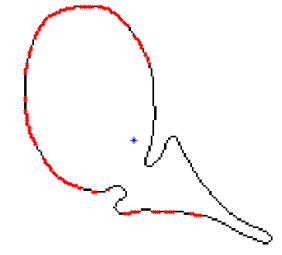

(d) All three constraints

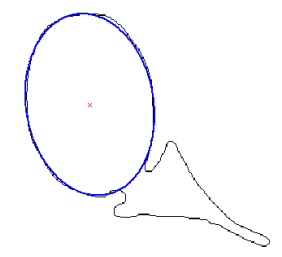

(e) Detected ellipse
Fig. 1: Sampling constraints: blue dot is the centroid and red points are pixel candidates, (b) SC ensures that selected points are locally smooth, (c) DC removes pixels that are too close to the centroid, (d) $\mathrm{CC}$ further removes outliers that do not belong to the target ellipse.

accuracy with 3 sampled points [6]. RHT is an instance of Monte Carlo methods [10] that rely on repeated random sampling.

Converging mapping in RHT means that the quality of sampled points is crucial for ellipse parameter estimation, which is not guaranteed due to its inherent uncertainty. Since in a complex/noisy image, the fraction of true ellipse pixels to foreground pixels is small, picking several pixels randomly will never narrow down to a right ellipse, as noted in [6]. This was addressed in [7] using iterative updates of the region enclosing the target ellipse but was not tested with multiple ellipses and interfering curves.

By imposing conditions and constraints on the sampled pixels we can improve accuracy and efficiency as described here. Connected components analysis is first performed; small components are eliminated since they are unlikely to be true foreground pixels that lie on the true ellipse. Each sample is taken from a single component since they have a higher probability of being on the same target ellipse. Constraints for smoothness, distance and curvature are described next with an example in Fig. 1.

Smoothness Constraint (SC): Each sampled point should be on a smooth portion of the edges for accurate estimation of ellipse parameters. We propose a measure of local smoothness on edge contours, Smoothness Index $(S)$. For a given foreground pixel $z, S(z)$ is defined as the standard deviation of gradient orientation of foreground pixels within the neighborhood of $z$, i.e., $S(z)=\operatorname{stdev}\left(\left\{\theta_{z^{\prime}} \mid z^{\prime} \in N_{z}\right\}\right)$, where $\theta_{z^{\prime}}$ is the gradient orientation (in radian) at pixel $z^{\prime}, N_{z}$ is the neighborhood of pixel $z$, a $m$-by- $m$ (set to 7) window centering at $z$. The selected pixels $\{z\}$ should satisfy

$$
s_{l} \leq S(z) \leq s_{u}
$$

where $s_{l}$ and $s_{u}$ are the lower/upper bounds of $S$, which are

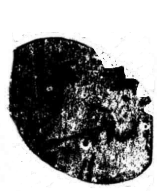

(a)

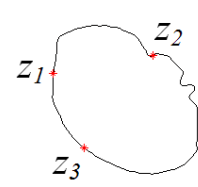

(b)

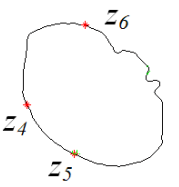

(c)

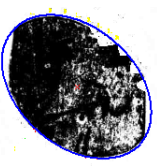

(d)
Fig. 2: Use of Smoothness Index $(S)$ to reject bad samples from random sampling. (a) An imperfect ellipse. (b) A bad sample on the edge, $S\left(z_{1}\right)=0.02, S\left(z_{2}\right)=0.45, S\left(z_{3}\right)=0.11$. (c) A good sample $\left(z_{4}, z_{5}, z_{6}\right)$ on the smooth portion of the edge where $S$ are 0.05, 0.07 and 0.21 respectively. (d) Ellipse detected by CRHT.

set to 0.03 and 0.25 empirically. Constrained sampling from edge pixels of a deformed ellipse is shown in Fig. 2.

Distance Constraint $(D C)$ : Given component properties: centroid (c), major axis length $\left(l_{1}\right)$ and minor axis length $\left(l_{2}\right)$ [11], the distance from sampled point $z$ to $\mathbf{c}, d_{z c}$, must satisfy

$$
r_{2} * l_{2} \leq d_{z c} \leq r_{1} * l_{1}
$$

where $r_{1}$ and $r_{2}$ are two preset thresholds.

Curvature Constraint (CC): Let $C$ be a plane curve, $P$ and $P^{\prime}$ be two neighbor points on $C$ shown in Fig. 3. The curvature $\kappa$ at $P$ is defined by $\kappa=\lim _{\delta s \rightarrow 0} \frac{\delta \boldsymbol{T}}{\delta s}$, which indicates that when $\delta s$ is sufficiently small, the direction of the curvature at $P$ can be approximated by the angle of $\delta \boldsymbol{T}$. Given a curve $C$ and a point $P$ on it (shown in Fig. 4(a)) where the curvature is non-zero, there is a unique circle that has the same tangent and curvature as curve $C$ at $P$, called the $o s$ culating circle at $P$. Given an ellipse $E$ and a point $P$ on it shown in Fig. 4(b), the center $O$ of its osculating circle at $P$ and the center $C$ of the ellipse must lie on the same side of the tangent $T$. To estimate the curvature direction at $P$, we consider its six closest neighbor points $P_{1}, \ldots, P_{6}$. The angle of the tangent at $P_{2}, \theta_{2}$, is approximated by $\theta_{2}=\frac{\theta_{P_{1}}+\theta_{P_{2}}+\theta_{P_{3}}}{3}$, where $\theta_{P_{i}}$ is the edge direction at $P_{i}$. Similarly, the angle of the tangent at $P_{5}, \theta_{5}=\frac{\theta_{P_{4}}+\theta_{P_{5}}+\theta_{P_{6}}}{3}$. Then the direction of the curvature $\kappa$ at point $P, \theta_{\kappa}(P)$, is approximated by $\theta_{\kappa}(P)=\tan ^{-1} \frac{\sin \theta_{5}-\sin \theta_{2}}{\cos \theta_{5}-\cos \theta_{2}}$. Since the angle between $\theta_{\kappa}(P)$ $(\overrightarrow{P O}$ ) and vector $\overrightarrow{P C}$ is usually below $\pi / 3$ (although it may be as large as $\frac{\pi}{2}$ ) a good ellipse pixel $P$ should satisfy

$$
\left|\theta_{\kappa}(P)-\theta_{\overrightarrow{P C}}\right| \leq \pi / 3
$$

\section{FITNESS MEASURE AND ELLIPSE VALIDATION}

Since the probability of a spurious ellipse is high, validation based on a robust fitness measure is essential. The Chamfer distance map [12], similarity \& distance [1] leads to a high false positive rate. We first identify true ellipse pixels using two constraints and then validate using weighted quality and completeness.

For an ellipse with parameters $(p, q, a, b, \theta)$, Eq. (1) may not hold due to low image resolution and edge distortion. A 


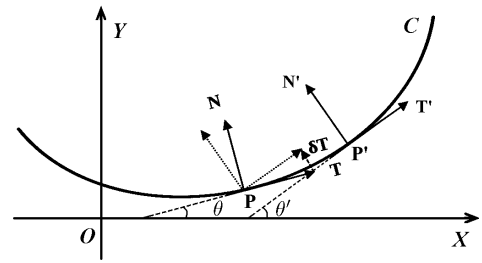

Fig. 3: The curvature of plane curves. $\boldsymbol{T}, \boldsymbol{T}^{\prime}$ are the unit tangent vectors at $P$ and $P^{\prime}$ respectively. $\boldsymbol{T}=[\cos \theta, \sin \theta], \boldsymbol{T}^{\prime}=$ $\left[\cos \theta^{\prime}, \sin \theta^{\prime}\right] . \delta \boldsymbol{T}=\boldsymbol{T}^{\prime}-\boldsymbol{T}=\left[\cos \theta^{\prime}-\cos \theta, \sin \theta^{\prime}-\sin \theta\right] . \delta s$ is the distance between the points.

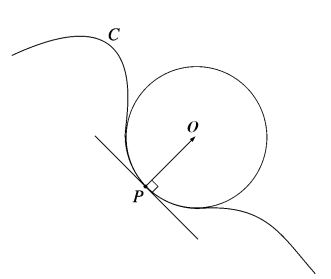

(a) Osculating circle at $P$

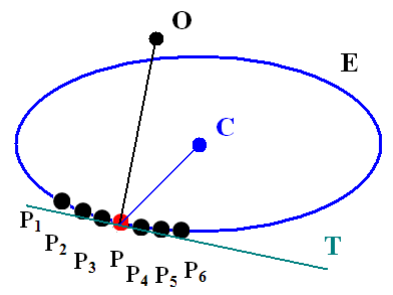

(b) curvature direction at $P$
Fig. 4: Curvature constraint. In (b), $C$ is the center of ellipse $E, T$ is the tangent at $P$, and $O$ is the center of the osculating circle at $P$.

relaxed constraint for pixels lying on a potential ellipse, $S_{v}=$ $\{(x, y)\}$, is

$$
\varepsilon_{(x, y)}=|f(x, y)-1| \leq \epsilon_{0},
$$

where $f(x, y)=\frac{((x-p) \cos \theta+(y-q) \sin \theta)^{2}}{a^{2}}+\frac{((y-q) \cos \theta-(x-p) \sin \theta)^{2}}{b^{2}}, \epsilon_{0}$ is a preset tolerance.

If the difference between the analytical derivative $d$ at each pixel $(x, y) \in S_{v}$, computed using Eq. (6), and the tangent of gradient orientation at $(x, y)$ is below tolerance $T_{1}$, then accept pixel $(x, y)$ as a true ellipse pixel, i.e.,

$d=\frac{\left(-\frac{2}{a^{2}}\right)\left[(x-p) \cos \theta \sin \theta+(y-q) \sin ^{2} \theta\right]+\left(-\frac{2}{b^{2}}\right)\left[(y-q) \cos ^{2} \theta-(x-p) \cos \theta \sin \theta\right]}{\left(-\frac{2}{a^{2}}\right)\left[(x-p) \cos ^{2} \theta+(y-q) \cos \theta \sin \theta\right]+\left(\frac{2}{b^{2}}\right)\left[(y-q) \cos \theta \sin \theta-(x-p) \sin ^{2} \theta\right]}$

$$
|d|_{(x, y)}-\tan \left(\left.\psi\right|_{(x, y)}\right) \mid \leq T_{1},
$$

where $\left.\psi\right|_{(x, y)}$ is the gradient orientation at $(x, y)$.

\subsection{Weighted Quality}

For a set of foreground pixels $\mathbf{z}=\left\{z_{i}=\left(x_{i}, y_{i}\right) \mid i=\right.$ $1, \ldots,|\mathbf{z}|\}$ associated with potential ellipse $E$ let $Z$ be a random variable that denotes the number of true ellipse pixels for ellipse $E, Z_{i}$ be a binary random variable representing the identity of pixel $z_{i}$ (i.e. $Z_{i}=1$ means pixel $z_{i}$ is a true ellipse pixel, $Z_{i}=0$ means otherwise). Suppose $Z_{i}$ and $Z_{j}$ are independent, $\forall i, j \in\{1, \ldots,|\mathbf{z}|\}, i \neq j$. Thus, $Z=\sum_{i=1}^{|\mathbf{z}|} Z_{i}$.

Assume a zero-mean Gaussian error distribution for a true ellipse pixel $z_{i}$, i.e., $P\left(\varepsilon_{z_{i}} \mid Z_{i}=1\right)=N\left(\varepsilon_{z_{i}} \mid 0, \sigma^{2}\right)$, where $\varepsilon_{z_{i}}$ is defined by Eq. (5), and $\sigma^{2}$ is the variance of $\varepsilon_{z_{i}}$.

Assuming no prior knowledge of the identity of pixel $z_{i}$ before we observe the fitting error $\varepsilon_{z_{i}}$, then $P\left(Z_{i}=1\right)\left(=P\left(Z_{i}=0\right)\right)$ is a constant. From Bayes' theorem

$$
\begin{aligned}
P\left(Z_{i}=1 \mid \varepsilon_{z_{i}}\right) & =P\left(Z_{i}=1\right) P\left(\varepsilon_{z_{i}} \mid Z_{i}=1\right) / P\left(\varepsilon_{z_{i}}\right) \\
& \propto P\left(\varepsilon_{z_{i}} \mid Z_{i}=1\right) .
\end{aligned}
$$

The expectation of $Z, \mathbb{E}[Z]$, is computed by $\mathbb{E}[Z]=$ $\sum_{i=1}^{|\mathbf{z}|} \mathbb{E}\left[Z_{i}\right]=\sum_{i=1}^{|\mathbf{z}|} P\left(Z_{i}=1 \mid \varepsilon_{z_{i}}\right)$. Then the weighted quality of ellipse $E, W Q_{E}$, is defined by

$$
W Q_{E}=\frac{\mathbb{E}[Z]}{c_{E}},
$$

where $c_{E}$, the circumference of ellipse $E$, is computed using Ramanujan's second approximation [13] $c_{E}=\pi(a+b)(1+$ $\left.\frac{3 h}{10+\sqrt{4-3 h}}\right)$, where $h=(a-b)^{2} /(a+b)^{2}$.

\subsection{Completeness}

To differentiate partial from full ellipses the distribution of pixels on the perimeter is used. The completeness $C_{E}=$ $\sigma_{E} / \sigma_{c}$ where $\sigma_{E}$ is the standard deviation of the angle in radians that all true ellipse pixels make with the center normalized by the standard deviation of angles distributed uniformly over $[0,2 \pi], \sigma_{c}=2 \pi / \sqrt{12} \approx 1.8138$.

\section{ALGORITHM CRHT}

1. For input grayscale image $\boldsymbol{I}$ compute edge map $\boldsymbol{b} \boldsymbol{w}$.

2. Decompose $\boldsymbol{b} \boldsymbol{w}$ into components, eliminate small ones, and initialize a global accumulator $A_{g}$.

3. For component $\hat{C}$, with properties $\mathbf{c}, l_{1}$ and $l_{2}$, initialize local accumulator $A_{l}$ and a weight $w$ to 0.5 .

4. Identify pixels $\hat{C}_{g} \subseteq \hat{C}$, which satisfy constraints given in Eqs. $(2,3,4)$, and initialize counter $c_{t}$ for the number of times searching for ellipse from $\hat{C}$ fails.

5. Draw a 3-pixel sample from $\hat{C}_{g}$, estimate center $(p, q)$ and compute $A, B$, and $C$ using approach in [6].

6. If $4 A C-B^{2} \leq 0$ increment $c_{t}$ by 1 and return to (5). Else convert parameters $(p, q, A, B, C)$ to its equivalent $(p, q, a, b, \theta)$ using: $\theta=0.5 * \cot ^{-1}((A-C) / B)$, $a=\left[1 /\left(A \cos ^{2} \theta+B \cos \theta \sin \theta+C \sin ^{2} \theta\right)\right]^{1 / 2}, b=$ $\left[1 /\left(A \sin ^{2} \theta-B \cos \theta \sin \theta+C \cos ^{2} \theta\right)\right]^{1 / 2}$.

7. Compute $r=\#$ of ellipse pixels/circumference.If $r<T_{2}$ (threshold for $r$ ), increment $c_{t}$ by 1 and go to (5). Otherwise, update $\mathbf{c}, l_{1}, l_{2}$ and $w$ using: $\mathbf{c}=\frac{\mathbf{c} * w+[p q] * r}{w+r}$, $l_{1}=\frac{l_{1} * w+2 a * r}{w+r}, l_{2}=\frac{l_{2} * w+2 b * r}{w+r}, w=\frac{w+r}{2}$. If any ellipse in $A_{l}$ has parameters close to $(p, q, a, b, \theta)$ then merge them and increase its score by 1 . Otherwise, insert into $A_{l}$ new entry $(p, q, a, b, \theta)$ with score 1 .

8. If $c_{t} \geq T_{3}$, a threshold for $c_{t}$, recompute $\hat{C}_{g}$ based on the latest value of $\mathbf{c}, l_{1}, l_{2}$. Repeat steps (4-8) until a preset number of samples for $\hat{C}$ have been tested. 
9. Select ellipse in $A_{l}$ with the highest score and put it into $A_{g}$. Repeat steps (3-9) for all components in $\boldsymbol{b} \boldsymbol{w}$.

10. Validate ellipse candidates in $A_{g}$ using criteria $W Q_{E} \geq$ $t_{Q}$ and $C_{E} \geq t_{C}$, where $t_{Q}$ and $t_{C}$ are thresholds.

\section{EXPERIMENTS AND RESULTS}

The CRHT algorithm, as well as RHT and MPGA, were evaluated with two data sets: 300 synthetic images (set $A$ ) and 500 images of footwear prints (set $B$ ). A sample image in each set in shown in Figs. 5 and 6. CRHT parameters were: $s_{l}=0.03, s_{u}=0.25, r_{1}=0.5, r_{2}=0.25, \epsilon_{0}=0.08$, $T_{1}=0.26, \sigma=0.08, T_{2}=0.4, T_{3}=15, t_{Q}=0.5, t_{C}=0.5$. Performance measures were: True/False Positive Rates (TPR $=N_{t} / N$ FPR $\left.=N_{f} / N\right)$ and mean accuracy MA $=S_{n} / S_{g}$, where $N$ ellipses are in the image, $N_{t}$ and $N_{f}$ are no. of ellipses detected, $S_{n}$ is the non-overlapping area of detected ellipse and ground truth, $S_{g}$ is area of ground truth ellipse. MA is measured only for correctly detected ellipses. Results in Table 1 show the high accuracy and efficiency of CRHT.

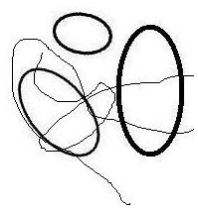

(a) Input

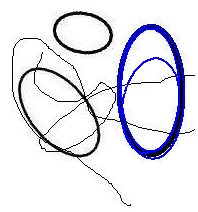

(b) RHT

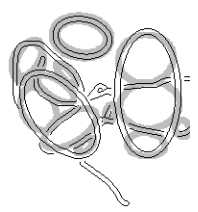

(c) MPGA

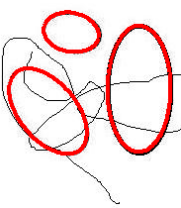

(d) Proposed
Fig. 5: Synthetic image: (a) input with 3 ellipses and noise lines, (b) RHT detects one ellipse, (c) MPGA detects one true ellipse and many spurious ones, and (d) CRHT detects only the three.

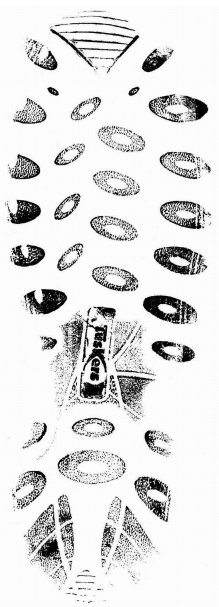

(a) Input

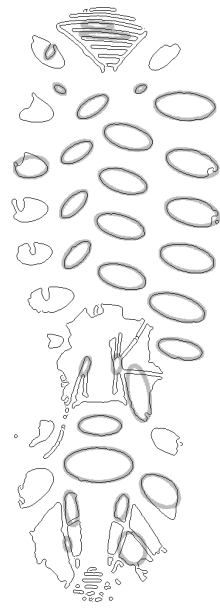

(b) MPGA

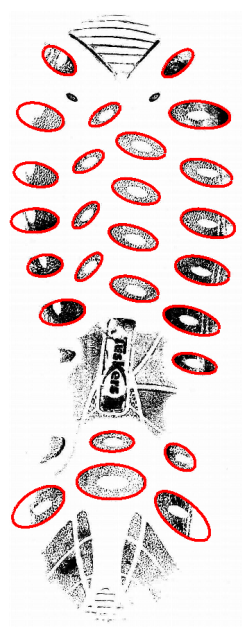

(c) Proposed
Fig. 6: Real image: (a) footwear impression with 16 full and 10 partial ellipses, (b) MPGA detects 16 full, 2 partials and spurious ones, and (c) CRHT performs correctly. RHT fails to detect any.

\begin{tabular}{|c|c|c|c|c|c|c|c|c|}
\hline \multirow{2}{*}{ Method } & \multicolumn{2}{|c|}{ TPR(\%) } & \multicolumn{2}{c|}{ FPR(\%) } & \multicolumn{2}{c|}{ MA } & \multicolumn{2}{c|}{ Time(s) } \\
\cline { 2 - 9 } & A & B & A & B & A & B & A & B \\
\hline RHT & 15.2 & 13.6 & 19.6 & 22.8 & 0.2 & - & 753.3 & 1046.5 \\
\hline MPGA & 30.6 & 28.4 & 38.8 & 42.4 & 0.1 & - & 2 & 5.3 \\
\hline CRHT & 98.3 & 97.5 & 0.02 & 0.02 & 0.04 & - & 5 & 6.5 \\
\hline
\end{tabular}

Table 1: Performance on datasets $A$ (synthetic) and $B$ (footwear prints). In case of MA with B ground truth was unavailable.

\section{SUMMARY AND CONCLUSIONS}

CRHT is a new Monte Carlo approach to ellipse detection which exploits constraints on sampled points. Incremental parameter learning guides the sampler to select high quality points for ellipse fitting. Two probabilistic fitness measures were introduced to validate extracted candidate ellipses. CRHT can detect multiple, potentially deformed, full (partial) ellipses in complex and noisy images. Accuracy and speed of the algorithm using both synthetic and real images is higher than RHT and MPGA.

\section{REFERENCES}

[1] J. Yao, N. Kharma, and P. Grogono, "A multi-population genetic algorithm for robust and fast ellipse detection," Pattern Anal. Appl., vol. 8, pp. 149-162, 2005.

[2] Z.Y. Liu and H. Qiao, "Multiple ellipses detection in noisy environments: a hierarchical approach," $P R, 2009$.

[3] Y. Tang, S. N. Srihari, and H. Kasiviswanathan, "Similarity and clustering of footwear prints," in Proc. Int. Conf. Granular Computing, 2010.

[4] R.O. Duda and P.E. Hart, "Use of Hough transform to detect lines and curves in pictures," Comm.ACM, 1972.

[5] L. Xu and E. Oja, "Randomized Hough transform (rht): basic mechanisms, algorithms, and computational complexities," CVGIP: Image Underst., 1993.

[6] R.A. McLaughlin, "Randomized Hough transform: Improved ellipse detection with comparison," Pattern Recognition Letters, 1998.

[7] W. Lu and J. Tan, "Detection of incomplete ellipse in images with strong noise by iterative randomized Hough transform (irht)," Pattern. Recog., vol. 41(4), 2008.

[8] F. Mai, Y.S. Hung, H. Zhong, and W.F. Sze, "A hierarchical approach for fast and robust ellipse extraction," Pattern Recog., vol. 41(8), pp. 2512 - 2524, 2008.

[9] K. Hahn, Y. Han, and H. Hahn, "Ellipse detection using a randomized Hough transform based on edge segment merging scheme," in Pro. Int. C. Sig. Proc. R. A., 2007.

[10] N. Metropolis and S. Ulam, "The Monte Carlo method," J. of the American Statistical Association, 1949. 
[11] http://www.mathworks.com/help/toolbox/ images/ref/regionprops.html.

[12] E. Lutton and P. Martinez, "A genetic algorithm for detection of $2 \mathrm{D}$ geometric primitives in images," in ICPR.

[13] S. Ramanujan, Ramanujan's Collected Works, Chelsea, New York, 1962. 